\title{
A
}

Acta HealthMedica

Acta HealthMedica (ISSN: 2414-6528)

http://www.ActaHealthMedica.com

Volume 2, Issue 2, April-June 2017, Pages: 174-175, DOI: http://dx.doi.org/10.19082/ah174

\section{ESTABLISHMENT OF NATIONAL SPINAL CORD AND COLUMN INJURY REGISTRY OF IRAN (NSCIR-IR): A PROSPECTIVE QUALITY REGISTRY}

Zahra Azadmanjir ${ }^{1}$, Bizhan Aarabi ${ }^{2}$, Mohammad Reza Zarei ${ }^{3}$, Khatereh Naghdi ${ }^{3}$, Seyed Behzad Jazayeri ${ }^{3}$, Mahdi Safdarian $^{3}$, Mahdi Sharif-Alhosseini ${ }^{3}$, Aidin Abedi $^{3}$, Jalil Arab Kheradmand ${ }^{4}$, Kazem Zendehdel ${ }^{5,6}$, Vafa RahimiMovaghar $^{3 *}$

1: Department of Health Information Management, School of Allied Medical Sciences, Tehran University of Medical Sciences, Tehran, Iran

2: Department of Neurosurgery, University of Maryland School of Medicine, Baltimore, Maryland, USA

3: Sina Trauma and Surgery Research Center, Tehran University of Medical Sciences, Tehran, Iran

4: Ahya Neuroscience Research Center, Tehran, Iran

5: Cancer Research Center, Cancer Institute of Iran, Tehran University Medical of Sciences, Tehran, I.R. Iran

6. Office of Disease and Health Outcome Registry, Deputy of Research and Technology, Ministry of Health and Medical Education, I.R Iran

\section{Correspondence:}

Vafa Rahimi-Movaghar, MD. Sina Trauma and Surgery Research Center, Tehran University of Medical Sciences, Tehran, Iran. Tel: +98216675002, Fax:+982166757009, E-mail: v_rahimi@yahoo.co

\section{TYPE OF ARTICLE: CONFERENCE ABSTRACT}

\begin{abstract}
Introduction: Spinal Cord Injury (SCI), a chronic and disabling condition, is often due to trauma. The annual incidence of SCI in the Iranian male and female population is an estimated 98.4 and 46.5 in 100,000 , respectively. There is a lack of reliable data source on the care and outcomes of individuals with SCI. On the other hand, policy-makers need reliable data to prevent SCI and improve its patient care and long-term outcomes. The aim of this project was to establish a prospective, registry that will be feasible in practice and provide the required data on etiology, level and type of injury, and quality of provided care, in addition to patients' outcomes and their quality of life.

Methods: The project was started in November 2014 by presenting the initial plan of registry and creating the steering committee consisting of major stakeholders. After approval of the project by the Ministry of Health, a multi-disciplinary expert panel, including 45 national and international specialists, contributed in determining patient eligibility criteria; case finding, registration, follow-up processes and minimum data set (MDS). MDS, which was developed based on International Spinal Cord Injury Data Set, includes 350 data items. In order to include iatrogenic SCI, all patients who are admitted to the neurosurgery departments of collaborating hospitals with acute spinal fracture/dislocation with or without SCI, were defined as the eligible patients for registry. Data sources and data collection methods were paper medical records, medical images, hospital information system, interview with patients and attending physician, and observation of patient health status. A web-based application was designed for our registry processes. To ensure the feasibility of registry, a seven-month pilot study was conducted with participation of two hospitals in Tehran and one in Rasht. The coverage rate of eligible cases, workload amount and time spent for registration and quality review were considered to evaluate the performance of registry.

Results: In the pilot phase, a total of 81 eligible patients were identified in three hospitals. Data of 16 patients from one hospital were not recorded into the electronic system. Thus, data of 65 patients were reviewed and verified. Data collection and quality control duration were $28 \pm 10$ and 15 minutes per patient, respectively. Duration of the whole process from beginning of data entry until final verification was nearly two weeks. The cause of the problem was shortage of trained quality reviewers. The mean
\end{abstract}

\section{Abstracts of First National Congress of Medical Informatics, Mashhad, Iran, February 2017}

(C) 2017 The Authors. This is an open access article under the terms of the Creative Commons Attribution-NonCommercialNoDerivs License, which permits use and distribution in any medium, provided the original work is properly cited, the use is non-commercial and no modifications or adaptations are made. 
coverage of registry was $65.36 \%$ due to lack of dedicated registrar and hidden spine fractures that were not diagnosed in admission time. Currently, 182 patients have been registered into the registry system up until 26 December, 2016. Of these, data of 114 patients have been verified, 49 patients are being completed and 19 are under quality review.

Conclusion: Although the implementation of NSCIR-IR registry is feasible, registry processes can be accelerated by assigning dedicated registrars and one physician to quality control in each registry center. Also, some modifications in eligibility criteria may be necessary for expanding the registry coverage.

Reinforcement of coordination between NSCIR-IR headquarters with registry centers outside of Tehran for timely registration seems necessary.

KEYWORDS: Registries, Feasibility studies, Quality of health care, Spinal cord injuries, Data collection 